\title{
Asymmetric redox status and cardiovascular risk factors in smoker women and men
}

\author{
Laura Celano $^{1,2}$, Anabel Vidal ${ }^{1}$, Alicia Olascoaga ${ }^{3}$, Walter Alallon ${ }^{3}$, Ana Denicola ${ }^{2,4}$, \\ Leonor Thomson ${ }^{1,2, *}$ \\ ${ }^{1}$ Enzimología, Facultad de Ciencias, Universidad de la República, Montevideo, Uruguay \\ ${ }^{2}$ Center for Free Radicals and Biomedical Research, CEINBIO, Universidad de la República, Montevideo, Uruguay \\ ${ }^{3}$ Laboratorio Clínico, Hospital de Clínicas, Facultad de Medicina, Universidad de la República, Montevideo, Uruguay \\ ${ }^{4}$ Fisicoquímica Biológica, Facultad de Ciencias, Universidad de la República, Montevideo, Uruguay
}

\section{Email address:}

1thomson@fcien.edu.uy (L. Thomson)

\section{To cite this article:}

Laura Celano, Anabel Vidal, Alicia Olascoaga, Walter Alallon, Ana Denicola, Leonor Thomson. Asymmetric Redox Status and Cardiovascular Risk Factors in Smoker Women and Men. American Journal of Clinical and Experimental Medicine.

Vol. 1, No. 3, 2013, pp. 53-57. doi: 10.11648/j.ajcem.20130103.13

\begin{abstract}
Profuse epidemiological evidence supports a higher risk for coronary heart disease and stroke in smoker women than in men with the same habit. Although it is already known that cigarette smoking alters the redox state, is unknown if the imbalance in the normal equilibrium between oxidants and antioxidants is responsible for the elevated female susceptibility. Therefore, the aim of this work was to estimate the effect of smoking on serum redox status in women compared with men, accounting for divergences in other major risk factors for cardiovascular disease. Lipid profile, antioxidant capacity, and serum carbonyls were assessed in 116 healthy Uruguayans, composed by 50 females and 66 males. The smoking habit was declared by 17 females (34\%) and 36 males $(54 \%)$. The lipid profile was modified by cigarette smoking, affecting in a different way males and females. In particular, HDL-C that was higher in non-smoker females (59 (28) $\mathrm{mg} / \mathrm{dL})$ than in non-smoker males (46 (14) $\mathrm{mg} / \mathrm{dL})$ significantly decreased in smoker females (51 (13) $\mathrm{mg} / \mathrm{dL}$ ), remaining unchanged in the smoker male population (42 (12) mg/dL). Conversely LDL-C, which gave similar values for non-smoker females $(110.1 \pm 35.2 \mathrm{mg} / \mathrm{dL})$ and males $(98.5 \pm 36.0 \mathrm{mg} / \mathrm{dL})$, increased exclusively in smoker males $(122.2 \pm 36.4 \mathrm{mg} / \mathrm{dL}, \mathrm{p}<0.05)$. In turn, the level of serum antioxidants that was higher in non-smoker males $(1.9 \pm$ $0.3 \mathrm{mM}$ FRAP) than females $(1.5 \pm 0.4 \mathrm{mM} /$ FRAP), remained unchanged in smokers. Similar results were obtained for carbonyls, which showed higher levels in non-smoker males $(0.90 \pm 0.32 \mathrm{nmol} / \mathrm{mg}$ of protein $)$ than females $(0.74 \pm 0.32$ $\mathrm{nmol} / \mathrm{mg}$ of protein), and while the level remained unmodified in smokers males $(0.86 \pm 0.28 \mathrm{nmol} / \mathrm{mg}$ of $\mathrm{protein})$ increased non-significantly in smoker females $(0.79 \pm 0.31 \mathrm{nmol} / \mathrm{mg}$ of protein). Whereas the oxidation index correlated positively with LDL-C $(\mathrm{r}=0.45)$ in smoker males, and with triglycerides in both non-smoker and smoker females $(\mathrm{r}=0.42$ and 0.79 , respectively), a negative and intense correlation with HDL-C $(\mathrm{r}=-0.79)$, and a positive one with the LDL-C/HDL-C index $(\mathrm{r}=0.87)$ was observed exclusively in smoker females. Our results point to an association at the molecular level between oxidative stress footprints and plasma lipoprotein/cholesterol concentration predominantly in smoker females, and support the concept that the higher sensitivity of women to smoking related cardiovascular pathology is associated with oxidants-mediated biomolecular insults.
\end{abstract}

Keywords: Cardiovascular Disease, Cigarette Smoking, Lipid Profile, Gender, Antioxidants, Protein Oxidation

\section{Introduction}

Several studies have demonstrated the participation of inflammation and oxidants mediated biomolecular damage in cardiovascular disease (CVD). [1]. While CVD is the main cause of death in aged populations, cigarette smoking is the primary cause of preventable morbidity and mortality
$[2,3]$. Smoking is a risk factor for cardiovascular disease and its prevalence is increasing in women mainly in low and middle income countries. Although premenopausal women have a less proatherogenic lipid profile than men and a lower incidence of cardiovascular events, an excess of risk for coronary artery disease independent of other risk factors in smoker females has been shown [4]. The 
underlying mechanisms by which smoking might be more hazardous in female smokers are unclear, and despite the fact that several causes have been suggested as culprits, including differential metabolism of cigarette toxins $[5,6]$ and the use of oral contraceptives [7], smoking still remained as an independent risk factor for CVD [4].

Differences in oxidative stress biomarkers in smoker women have been reported. Between them, greater levels of 8-hydroxy-2'-deoxyguanosine (8OHdG) and 8 -iso-prostaglandin F2 $\alpha$ were described in female smokers, being this difference significant only in current smokers [8]. Urinary 8-OHdG was also augmented in hypertensive smoker women, showing an important correlation with plasma indexes of inflammation, in particular TNF- $\alpha$, sTNF-R1, sTNF-R2, and IL-6 [9]. In a recent study a significant increase in the level of homocysteine and the endogenous nitric oxide synthase inhibitor asymmetric dimethylarginine (ADMA), both involved in vascular inflammation and endothelial dysfunction [10], were reported in smoker females [11]. Moreover, in a five year follow up, the association of greater oxidative stress, as indicated by elevated serum protein carbonyls, with a higher risk of death in older women was reported [12]. Those evidences point out to a higher susceptibility in the female population against oxidants generated during tobacco burning. Additionally, due to the high prevalence of subclinical manifestations of coronary atherosclerosis in women, most clinical protocols and epidemiological studies under-estimate the female risk [13], that together with the lack of diagnostic tools to properly estimate the risk before the onset of irreversible pathological outcomes [11], impose the need of developing new diagnostic approaches specially designed for women with high risk of CVD.

We hypothesize that women at high risk of CVD because of being smokers, and despite the fact of having a low risk lipid profile, will have a disturbed redox stratus and its evaluation will help to make earlier therapeutic decisions. Protein carbonyls, which are a footprint of oxidative protein attack [14, 15], and the measurement of antioxidants by the ferric reducing power of plasma (FRAP) [16] were chosen, because both techniques are relatively simple and inexpensive, and accordingly suitable for application in clinical laboratories with low levels of complexity.

\section{Materials and Methods}

Human subjects: Blood donors (116) assisting to the Hemotherapy Department of Hospital de Clínicas were voluntary enrolled in this study. All participants were healthy, non-reporting previous cardiovascular, hepatic, gastrointestinal, or renal diseases. None of the participants was taken medications or vitamin supplements, and the exclusion criteria was the same as for blood donation, including hypertension, diabetes, history of any type of CVD, or recent infections. Participants were properly informed and asked to sign a written consent, and the study protocol was approved by the institutional review board of the hospital. Blood was taken by antecubital venipuncture after $12 \mathrm{~h}$ of starvation in serum tubes with gel (Vacuette, Grainer-Bio Spain). Serum samples were processed and assayed immediately or stored at $-20^{\circ} \mathrm{C}$ in aliquots until use (less than a week). All cases were interviewed using a questionnaire, which included data on smoking, alcohol and "mate" (common local beverage) consumption, nutritional habits, height, and weight. BMI was calculated.

Serum chemistry. Total cholesterol (TC), triglycerides (TG), and high density lipoprotein-cholesterol (HDL-C) were determined by enzymatic procedures, and low density lipoprotein-cholesterol (LDL-C) was estimated using the Friedewald equation: LDL-C=TC-(HDL-C+TG/5) [18]. Uric acid was determined enzymatically. Bilirubin was determined by the diazo reaction and total proteins were determined by the Biuret method. All the reactions were performed with reagents, controls and calibrators from Roche Diagnostics, using a Hitachi 911 (Roche Diagnostics, Basel, Switzerland).

Serum antioxidants. The antioxidant content of serum was evaluated by the ferric reducing ability of plasma (FRAP) as described [16, 17]. For calibration, aqueous solutions of $0.25-1 \quad \mathrm{mM}$ 6-hydroxy-2,5,7,8tetramethylchroman-2-carboxylic acid (Trolox, Sigma Aldrich, St. Louis, MO) were used. The reaction proceeded at $37^{\circ} \mathrm{C}$ for $4 \mathrm{~min}$ and the absorbance of the $\mathrm{Fe}^{2+}$-2,4-tripyridyl-s-triazine (TPTZ, Sigma-Aldrich, St. Luis, MO) complex was measured at $593 \mathrm{~nm}$ using a Shimadzu UV-1603 spectrophotometer.

Protein carbonyls. Serum carbonyl content was determined using the 2,4-dinitrophenylhydrazine (DNPH) method [19]. Briefly, proteins were precipitated with trichloroacetic acid (1.8\%) in ice, after 3 min the samples were centrifuged at $13000 \mathrm{~g}$ for $3 \mathrm{~min}$. The pellet was dissolved in $10 \mathrm{mM}$ DNPH (Sigma Aldrich, St. Louis, MO) in $2 \mathrm{M} \mathrm{HCl}$, and incubated for 1 hour at room temperature with occasional vortexing. After incubation, the remaining reagent was removed by protein precipitation in the same conditions, and the pellet was suspended in $6 \mathrm{M}$ guanidine at $37^{\circ} \mathrm{C}$. Any insoluble material was removed by additional centrifugation. Absorbance was read using a Varian Cary 50 spectrophotometer (Agilent Technologies, Australia). Carbonyl content was calculated from the difference in absorbance at $360 \mathrm{~nm}-390 \mathrm{~nm}\left(\varepsilon=22 \mathrm{mM}^{-1} \mathrm{~cm}^{-1}\right)$ [20]. The results were expressed as nmol/mg of protein. Total protein concentration was determined using the Bradford method.

Statistics. Statistical evaluation was carried out using OriginPro 8 SRO (OriginLabCorporation, Northampton, MA, USA). Values were expressed as mean and standard deviation. Differences between groups were calculated by one way ANOVA (considering significant differences with $\mathrm{p}<0.05$ ), followed by the Bonferroni test. Since some of the variables (bilirubin, TG and HDL-C) failed to prove a normal distribution by the Kolmogorov-Smirnov test, these 
Table 1. Population profile

\begin{tabular}{|c|c|c|c|c|}
\hline \multirow[b]{2}{*}{ Parameter } & \multicolumn{2}{|l|}{ Males } & \multicolumn{2}{|l|}{ Females } \\
\hline & $\begin{array}{l}\text { Non-Smokers } \\
(\mathrm{n}=30)\end{array}$ & $\begin{array}{l}\text { Smokers } \\
(\mathrm{n}=36)\end{array}$ & $\begin{array}{l}\text { Non-Smokers } \\
(\mathrm{n}=33)\end{array}$ & $\begin{array}{l}\text { Smokers } \\
(\mathrm{n}=17)\end{array}$ \\
\hline Age (years) & $32.8 \pm 10.2$ & $37.4 \pm 10.8$ & $35.9 \pm 12.4$ & $30.5 \pm 7.2$ \\
\hline $\operatorname{BMI}\left(\mathrm{Kg} / \mathrm{m}^{2}\right)$ & $26.7 \pm 3.6$ & $27.3 \pm 3.6$ & $24.5 \pm 4.6$ & $24.3 \pm 2.5^{\mathrm{b}}$ \\
\hline Proteins $(\mathrm{g} / \mathrm{dL})$ & $7.2 \pm 0.5$ & $6.9 \pm 0.5$ & $7.1 \pm 0.6$ & $6.9 \pm 0.6$ \\
\hline Bilirubin $(\mathrm{mg} / \mathrm{dL})$ & $0.72(0.28)$ & $0.64(0.35)$ & $0.47(0.17)^{\mathrm{b}}$ & $0.50(0.17)^{\mathrm{c}}$ \\
\hline Uric acid (mg/dL) & $5.0 \pm 1.5$ & $5.4 \pm 1.6$ & $3.6 \pm 1.1^{\mathrm{b}}$ & $4.0 \pm 1.4^{\mathrm{c}}$ \\
\hline FRAP (mM) & $1.9 \pm 0.3$ & $1.7 \pm 0.4$ & $1.5 \pm 0.4^{b}$ & $1.6 \pm 0.4$ \\
\hline $\mathrm{TG}(\mathrm{mg} / \mathrm{dL})$ & $96(77)$ & $108(55)$ & $88(67)$ & $69(70)$ \\
\hline $\mathrm{TC}(\mathrm{mg} / \mathrm{dL})$ & $171.4 \pm 44.5$ & $188.4 \pm 42.1$ & $185.3 \pm 41.1$ & $178.1 \pm 43.5$ \\
\hline LDL-C (mg/dL) & $98.5 \pm 36.0$ & $122.2 \pm 36.4^{\mathrm{a}}$ & $110.1 \pm 35.2$ & $101.2 \pm 35.9$ \\
\hline HDL-C (mg/dL) & $46(14)$ & $42(12)$ & $59(28)^{b}$ & $51(13)^{\mathrm{a}}$ \\
\hline LDL-C/HDL-C & $3.9 \pm 1.5$ & $4.6 \pm 1.4$ & $3.4 \pm 0.9$ & $3.7 \pm 1.6$ \\
\hline
\end{tabular}

Values are expressed as mean $\pm \mathrm{SD}$, and were analyzed by one way ANOVA and the Bonferroni test. Since a normal distribution could not be proven for bilirubin, triglycerides and HDL-C by the Kolmogorov-Smirnov test, values were expressed as median and (IQR), and differences calculated by the Kruskal-Wallis test. Statistically significant differences $(\mathrm{p}<0.05)$ between non-smokers and smokers of the same sex ${ }^{\mathrm{a}}$, and between genders in non-smokers ${ }^{\text {, and smokers }}$, are shown.

values were expressed as medians and interquartile range (IQR). In the last case the Kruskal-Wallis test was employed to compare parameters between sex and the smoking habit, considering significant differences with $\mathrm{p}<0.05$. Boxes and whiskers were used to represent means and associated percentiles. Correlations were analyzed by Spearman's rank correlation coefficient, accepting correlations with $r \geq 0.5$ and $p<0.01$ as significant.

\section{Results}

Serum from 116 healthy blood donors that voluntarily assisted to the Hemotherapy Department of Hospital de Clínicas was collected. Fifty (43\%) participants were females, and $66(57 \%)$ were males. The mean age was 34.8 \pm 10.9 years, without differences between females $(34.1 \pm$ 11.1 years) and males (35.3 \pm 10 years). Almost half of the donors were smokers $(45.7 \%)$, being this habit more frequent in males $(54.5 \%)$ than in females $(34 \%)$. Most of the interviewed (89\%) drank "mate" and a $100 \%$ described a diet reach in red meat, both popular customs in the country. Concurrently with lower levels of uric acid $(5.0 \pm 1.5$ and $3.6 \pm 1.1 \mathrm{mg} / \mathrm{dL}$, in non-smoker males and females, respectively) and bilirubin $(0.72(0.28)$ and 0.47 $(0.17) \mathrm{mg} / \mathrm{dL}$ in non-smoker males and females, respectively) non-smoker females showed a lower level of antioxidants $(1.5 \pm 0.4 \mathrm{mM}$ FRAP) as compare with non-smoker males $(1.9 \pm 0.3 \mathrm{mM}$ FRAP). Although uric acid $(5.4 \pm 1.6$ and $4.0 \pm 1.4 \mathrm{mg} / \mathrm{dL}$ in smoker males and females, respectively) and bilirubin $(0.64(0.35)$ and $0.50(0.17)$ in smoker males and females, respectively) remained significantly lower in smoker females $(1.7 \pm 0.4$
mM FRAP), the antioxidant level decreased in the smoker male population approaching to smoker female values (1.6 \pm 0.4 mM FRAP) (Table 1 ).

The smoking habit also showed a differential effect on the lipid profile of males and females (Table 1). In males, the LDL-C concentration increased significantly $(\mathrm{p}<0.05)$ from $98.5 \pm 36.0 \mathrm{mg} / \mathrm{dL}$ in non-smokers to $122.2 \pm 36.4$ $\mathrm{mg} / \mathrm{dL}$ in smokers, while in smoker females this parameter remained unchanged $(110.1 \pm 35.2 \mathrm{mg} / \mathrm{dL}$ and $101.2 \pm 35.9$ $\mathrm{mg} / \mathrm{dL}$ in non-smokers and smoker females, respectively). Conversely HDL-C, which was significantly higher $(\mathrm{p}<0.05)$ in non-smoker females $(59(28) \mathrm{mg} / \mathrm{dL})$ than in non-smoker males (46 (14) $\mathrm{mg} / \mathrm{dL}$ ), decreased exclusively in smoker females to $51(13) \mathrm{mg} / \mathrm{dL}$ (Table 1). No statistically significant differences by gender were obtained for TG (96 (77) and 88 (67) mg/dL for non-smoker males and females, respectively), TC (171.4 $\pm 44.5 \mathrm{mg} / \mathrm{dL}$ and $185.3 \pm 41.1 \mathrm{mg} / \mathrm{dL}$, for non-smoker males and females, respectively), and the LDL-C/HDL-C index (3.9 \pm 1.5 and $3.4 \pm 0.9$ in non-smoker males and females, respectively), and no changes induced by the smoking habit were observed in these parameters (Table 1).

Serum carbonyls, analyzed as a parameter for cumulative protein oxidation, were significantly higher in non-smoker males $(0.90 \pm 0.32 \mathrm{nmol} / \mathrm{mg}$ of protein $)$ than the same group of females $(0.74 \pm 0.32 \mathrm{nmol} / \mathrm{mg}$ of protein). Changes in serum carbonyls in smoker females $(0.79 \pm$ $0.31 \mathrm{nmol} / \mathrm{mg}$ of protein $)$ and males $(0.86 \pm 0.28 \mathrm{nmol} / \mathrm{mg}$ of protein) were not significant (Fig. 1). Despite the lack of effect of the smoking habit on plasma carbonyls, several components of the lipid profile correlated with this parameter, mostly in smokers. In fact, a strong correlation 
between serum carbonyls and LDL-C $(r=0.45, \mathrm{p}<0.01)$ was seen in smoker males, while this relation was absent in non-smoker males or females without or with the habit. On the other hand, in smoker females carbonyls showed a significant negative correlation with HDL-C (-0.70, $\mathrm{p}<0.01$ ), and a positive one with the LDL-C/HDL-C ratio $(0.66, \mathrm{p}<0.01)$ (Table 2). On the contrary, TC correlated with carbonyls $(r=0.49, p<0.01)$ in non-smoker females. Whereas carbonyls remained un-related to age in both sexes; in non-smoker females a correlation was found for uric acid, probably due again to the redox properties of this compound.

Table 2. Influence of several parameters on serum carbonyls

\begin{tabular}{lllll}
\hline \multirow{2}{*}{ Parameter } & Males & \multicolumn{3}{l}{ Females } \\
& Non-Smokers & Smokers & Non-Smokers & Smokers \\
\hline & $\mathbf{r}_{\mathbf{s}}$ & $\mathbf{r}_{\mathbf{s}}$ & $\mathbf{r}_{\mathbf{s}}$ & $\mathbf{r}_{\mathbf{s}}$ \\
Age & 0.29 & 0.34 & -0.02 & -0.06 \\
BMI & 0.09 & -0.15 & 0.13 & 0.18 \\
Proteins & 0.13 & -0.38 & 0.16 & -0.2 \\
Bilirubin & 0.06 & -0.35 & 0.13 & 0.14 \\
Uric acid & 0.22 & -0.18 & $0.59^{\mathrm{a}}$ & 0.28 \\
FRAP & 0.005 & -0.20 & 0.19 & 0.39 \\
TG & -0.16 & 0.03 & 0.42 & 0.42 \\
TC & -0.08 & -0.40 & $0.49^{\mathrm{a}}$ & 0.32 \\
LDL-C & -0.03 & $-0.45^{\mathrm{a}}$ & 0.43 & 0.48 \\
HDL-C & -0.07 & 0.04 & 0.08 & $-0.70^{\mathrm{a}}$ \\
LDL-C/HDL-C & 0.02 & -0.19 & 0.28 & $0.66^{\mathrm{a}}$ \\
\hline
\end{tabular}

Spearman rank correlation coefficients $\left(\mathrm{r}_{\mathrm{s}}\right)$ for the stated parameters against carbonyl concentrations were calculated. Significance levels $\left({ }^{\mathrm{a}} \mathrm{p}<\right.$ 0.01 ) are reported.

\section{Conclusions}

The aim of this work was to explore the influence of gender and the smoking habit on serum indexes of oxidative stress, and the relationship of these indexes with well characterized risk factors for CVD. To pursue this objective, serum protein carbonyls and total antioxidants (FRAP) were measured, together with an evaluation of the lipid profile. Neither a decrease of the antioxidant level nor an increase of the oxidative stress index was induced by cigarette smoking. However, significant differences between genders were found in FRAP values as well as in carbonyls. Smaller FRAP values in women coincided with reduced serum concentrations of uric acid and bilirubin, as shown in Table 1, in accordance with the determinant role of both compounds as redox modulators [21].

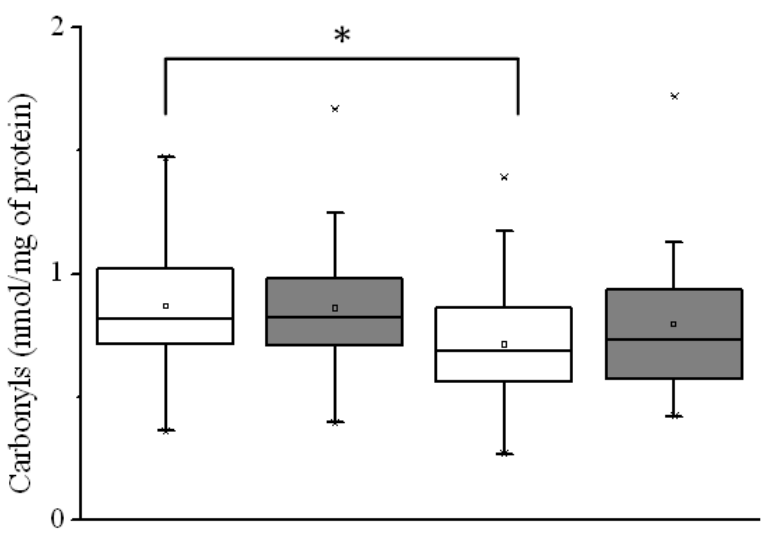

\section{Males}

Females

Figure 1. Influence of gender and the smoking habit on serum carbonyls. Protein carbonyls were evaluated in serum from 30 and 33 non-smoker males and females, respectively (white bars), 36 and 17 smoker males and females, respectively (grey bars). Box-whisker plots encompass the 25th and 75 th percentiles and lines within boxes represent median values. Bars represent the 5th and 95th percentiles. Statistically significant differences were found between non-smoker males and females (*, $p<0.05)$.

Carbonyls also were higher in non-smoker males than in non-smoker females, and showed significant positive correlations with TC and the LDL-C/HDL-C ratio, and a negative one with HDL-C (Table 2). Those correlations showed a distinctive gender pattern in agreement with a growing body of evidence about the dimorphism in CVD risk factors and outcomes that exist between females and males [22, 23]. For instance, TG was reported as a better predictor of CVD risk in women, whereas LDL-C concentration was a stronger predictor in men [24]. Actually, men with a higher risk for CVD, because of being smokers, showed an important increase on LDL-C serum concentration and this parameter correlated significantly with carbonyls. Conversely, in women, a correlation of protein carbonyls and TC emerged also in the absence of the smoking habit. Importantly, the HDL-C level, which decreased in smoker females, also correlated negatively with carbonyls in this group. In concordance with our results, a gender-specific correlation between plasma myeloperoxidase levels and serum high-density lipoprotein-associated paraoxonase-1 levels was reported recently in patients with coronary artery disease [25]. The connections found between serum lipids and carbonyls may be just the association of independent factors reflecting an increased risk for CVD in smokers, or may reflect a more close association involving a higher oxidation level of amino acid residues in apolipoproteins and potential existence of protein adducts with lipid alkenals [26].

In summary, although some limitations due to sample size and the necessity of a better characterization of the source of the oxidation products measured, this study contributes to remark the interplay between oxidative stress and classical risk factors for CVD, and also to the already recognized inter-gender differences about the susceptibility to tobacco smoke. 


\section{Acknowledgements}

We gratefully appreciate the contribution of the participants and staff of the Hemotherapy Department of Hospital de Clínicas, in particular, Professor Antonio Arago. This research was partially supported by Comisión Sectorial de Investigaciones Científicas (CSIC), Universidad de la República and PEDECIBA. Laura Celano was a fellow of Agencia Nacional de Investigación e Innovación (ANII).

\section{References}

[1] Stephens, J. W.; Gable, D. R.; Hurel, S. J.; Miller, G. J.; Cooper, J. A.; Humphries, S. E. Increased plasma markers of oxidative stress are associated with coronary heart disease in males with diabetes mellitus and with 10-year risk in a prospective sample of males. Clin Chem 52:446-452; 2006.

[2] Wang, J. C.; Bennett, M. Aging and atherosclerosis: mechanisms, functional consequences, and potential therapeutics for cellular senescence. Circ Res 111:245-259; 2012.

[3] Tobacco. Fact sheet No 339. WHO Media Centre; 2013.

[4] Huxley, R. R.; Woodward, M. Cigarette smoking as a risk factor for coronary heart disease in women compared with men: a systematic review and meta-analysis of prospective cohort studies. Lancet 378:1297-1305; 2011.

[5] Woodward, M.; Tunstall-Pedoe, H.; Smith, W. C.; Tavendale, R. Smoking characteristics and inhalation biochemistry in the Scottish population. J Clin Epidemiol 44:1405-1410; 1991.

[6] Parish, S.; Collins, R.; Peto, R.; Youngman, L.; Barton, J.; Jayne, K.; et al. Cigarette smoking, tar yields, and non-fatal myocardial infarction: 14,000 cases and 32,000 controls in the United Kingdom. The International Studies of Infarct Survival (ISIS) Collaborators. BMJ 311:471-477; 1995.

[7] McClave, A. K.; Hogue, C. J.; Brunner Huber, L. R.; Ehrlich, A. C. Cigarette smoking women of reproductive age who use oral contraceptives: results from the 2002 and 2004 behavioral risk factor surveillance systems. Women Health Issues 20:380-385; 2010.

[8] Hakim, I. A.; Harris, R.; Garland, L.; Cordova, C. A.; Mikhael, D. M.; Sherry Chow, H. H. Gender difference in systemic oxidative stress and antioxidant capacity in current and former heavy smokers. Cancer Epidemiol Biomarkers Prev 21:2193-2200; 2012.

[9] Rosello-Lleti, E.; de Burgos, F. G.; Morillas, P.; Cortes, R.; Martinez-Dolz, L.; Almenar, L.; et al. Impact of cardiovascular risk factors and inflammatory status on urinary 8-OHdG in essential hypertension. Am J Hypertens $25: 236-242 ; 2012$.

[10] Dayal, S.; Lentz, S. R. ADMA and hyperhomocysteinemia. Vasc Med 10 Suppl 1:S27-33; 2005.

[11] Campesi, I.; Carru, C.; Zinellu, A.; Occhioni, S.; Sanna, M.; Palermo, M.; et al. Regular cigarette smoking influences the transsulfuration pathway, endothelial function, and inflammation biomarkers in a sex-gender specific manner in healthy young humans. Am J Transl Res 5:497-509; 2013.

[12] Semba, R. D.; Ferrucci, L.; Sun, K.; Walston, J.; Varadhan, R.; Guralnik, J. M.; et al. Oxidative stress is associated with greater mortality in older women living in the community. J Am Geriatr Soc 55:1421-1425; 2007.

[13] Michos, E. D.; Vasamreddy, C. R.; Becker, D. M.; Yanek, L. R.; Moy, T. F.; Fishman, E. K.; et al. Women with a low Framingham risk score and a family history of premature coronary heart disease have a high prevalence of subclinical coronary atherosclerosis. Am Heart J 150:1276-1281; 2005.

[14] Beal, M. F. Oxidatively modified proteins in aging and disease. Free Radic Biol Med 32:797-803; 2002.

[15] Chevion, M.; Berenshtein, E.; Stadtman, E. R. Human studies related to protein oxidation: protein carbonyl content as a marker of damage. Free Radic Res 33 Suppl:S99-108; 2000.

[16] Tomasina, F.; Carabio, C.; Celano, L.; Thomson, L. Analysis of two methods to evaluate antioxidants. Biochemistry and molecular biology education: Biochem Mol Biol Educ 40:266-270; 2012.

[17] Benzie, I. F.; Strain, J. J. The ferric reducing ability of plasma (FRAP) as a measure of "antioxidant power": the FRAP assay. Anal Biochem 239:70-76; 1996.

[18] Friedewald, W. T.; Levy, R. I.; Fredrickson, D. S. Estimation of the concentration of low-density lipoprotein cholesterol in plasma, without use of the preparative ultracentrifuge. Clin Chem 18:499-502; 1972.

[19] Fields, R.; Dixon, H. B. Micro method for determination of reactive carbonyl groups in proteins and peptides, using 2,4-dinitrophenylhydrazine. Biochem J 121:587-589; 1971.

[20] Levine, R. L.; Garland, D.; Oliver, C. N.; Amici, A.; Climent, I.; Lenz, A. G.; Ahn, B. W.; Shaltiel, S.; Stadtman, E. R. Determination of carbonyl content in oxidatively modified proteins. Methods Enzymol 186:464-478; 1990.

[21] Huang, D.; Ou, B.; Prior, R. L. The chemistry behind antioxidant capacity assays. J Agric Food Chem 53:1841-1856; 2005.

[22] Konhilas, J. P. What we know and do not know about sex and cardiac disease. J Biomed Biotechnol 2010:562051; 2010 .

[23] Meagher, E. A. Addressing cardiovascular disease in women: focus on dyslipidemia. J Am Board Fam Pract $17: 424-437 ; 2004$.

[24] Mittendorfer, B. Sexual dimorphism in human lipid metabolism. J Nutr 135:681-686; 2005.

[25] Yunoki, K.; Naruko, T.; Inaba, M.; Inoue, T.; Nakagawa, M.; Sugioka, K.; et al. Gender-specific correlation between plasma myeloperoxidase levels and serum high-density lipoprotein-associated paraoxonase-1 levels in patients with stable and unstable coronary artery disease. Atherosclerosis 231:308-314, 2013.

[26] Domingues RM, Domingues P, Melo T, Pérez-Sala D, Reis A, Spickett CM Lipoxidation adducts with peptides and proteins: Deleterious modifications or signaling mechanisms? J Proteomics. 92:110-31; 2013. 